\title{
Analysis of the Factors Affecting Surgical Site Infection and Bone Flap Resorption after Cranioplasty with Autologous Cryopreserved Bone: The Importance of Temporalis Muscle Preservation
}

\author{
Sung-Won JIN¹, Sang-Dae KIM¹, Sung-Kon HA¹, Dong-Jun LIM¹', Hwa LEE², Hi-Jin YOU³ \\ ${ }^{1}$ Korea University, Ansan Hospital, Department of Neurosurgery, Ansan, Gyeonggi-do, Republic of Korea \\ ${ }^{2}$ Korea University, Ansan Hospital, Department of Ophthalmology, Ansan, Gyeonggi-do, Republic of Korea \\ ${ }^{3}$ Korea University, Ansan Hospital, Department of Plastic and Reconstructive Surgery, Ansan, Gyeonggi-do, Republic of Korea
}

\section{ABSTRACT}

AIM: To investigate the outcomes and associated complications after delayed cranioplasty using autologous, cryopreserved bone. MATERIAL and METHODS: This retrospective study included 57 consecutive patients treated with cranioplasty with autologous cryopreserved bone for various conditions causing increased intracranial pressure due to brain swelling. The incidence and risk factor of surgical site infection (SSI) and bone flap resorption were analyzed.

RESULTS: The SSI rate was $12.3 \%$ and the bone flap resorption rate was $24.0 \%$. There were statistically significant differences in SSI rate in relation to time from craniectomy to cranioplasty $(p=0.002)$ and previous temporalis muscle resection $(p=0.021)$. These factors were also independently associated with surgical site infection (time from craniectomy to cranioplasty: OR 0.901, 95\% $\mathrm{Cl}$ 0.826-0.982, $\mathrm{p}=0.018$; previous temporalis muscle resection: OR 11.607, 95\% Cl 1.155-116.590, $\mathrm{p}=0.037$ ). There was also a statistically significant difference in the bone flap resorption rate in relation to previous temporalis muscle resection ( $p=0.001)$. This factor was associated with bone flap resorption (OR 11.667, 95\% Cl 2.276-59.798, p=0.003).

CONCLUSION: The risk of these complications particularly increased after previous temporalis muscle resection. Based on this finding, we believe that preservation of the temporalis muscle may help to decrease postoperative complications after autologous cranioplasty.

KEYWORDS: Bone resorption, Cranioplasty, Cryopreservation, Decompressive craniectomy, Surgical site infection

\section{INTRODUCTION}

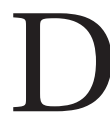
ecompressive craniectomy is a standard and important surgical method for refractory intracranial hypertension patients with conditions such as malignant cerebral edema, brain herniation due to cerebral infarction, intracranial hemorrhage and severe traumatic brain injury $(9,21,27)$.

After decompressive craniectomy, cranioplasty is necessary to remove physical and psychological burdens for patients due to skull defects, protect brain tissue and restore intracranial pressure (18). The use of autologous cryopreserved bone in cranioplasty can help avoid an immune response when using artificial patch material. Autologous cryopreserved bone is often preferred to artificial patch material because it is perfectly matched to the bony defect and reduces healthcare costs $(12,18,28)$.

Despite these advantages, complication rates for cranioplasty with autologous cryopreserved bone are known to be higher 
than complication rates for other neurosurgical surgeries. In particular, surgical site infection (SSI) and bone flap resorption are the most feared complications that require surgical removal of the implanted bone (25).

This retrospective study was conducted to identify complications after cranioplasty with autologous cryopreserved bone and to search for possible risk factors associated with the development of SSI and bone flap resorption.

\section{MATERIAL and METHODS}

\section{Patient Population}

This retrospective study included 57 patients treated with cranioplasty with autologous cryopreserved bone from May 2006 to October 2014 for various conditions causing increased intracranial pressure due to brain swelling. We excluded patients who underwent decompressive craniectomy at another hospital or cranioplasty combined with another operation, as well as patients with a SSI after craniectomy and traumatic brain injury with an open dirty wound at the scalp. Medical records and radiologic imaging studies were reviewed and clinical data were collected.

Analysis factors were age, gender, medical co-morbidities (diabetes mellitus, smoking), causes of craniectomy, the time from craniectomy to cranioplasty, operation time, bilateral cranioplasty and previous temporalis muscle resection.

SSI was defined as a surgical wound site that exhibited focal erythema, pus-like discharge or wound rupture (4). Bone flap resorption was defined as a remnant thickness of the bone flap edge that was less than $50 \%$ compared to the thickness on a brain computed tomography (CT) scan taken immediately after cranioplasty and follow-up brain CT (Figure 1A, B). The brain CT data were recorded by two neurosurgeons.

\section{Storage Method}

After decompressive craniectomy, the soft tissue attached to the bone flap was completely removed, sufficiently irrigated with normal saline and then packed in two layers of sterile plastic bags. The bone flaps were stored in our bone bank at a temperature of $-70^{\circ} \mathrm{C}$ within 30 minutes.

\section{Cranioplasty Procedure}

On the day of the cranioplasty, the bone flap was taken out of the bone bank at the beginning of the operation. The bone flap was soaked in a betadine solution for 30 minutes prior to implantation.

A skin incision was made at the previous scar, and the temporalis muscle was carefully dissected from the dura, avoiding any cerebrospinal fluid leak. The periosteum was cut open and separated at the edge of the bone window to completely expose the bone window edge. The bone was then put into place and fixed to the skull using a skull lock or titanium plating system. The temporalis muscle was reattached to the temporal bone. In cases where the muscle was adherent and difficult to release from the dura, the bone flap was replaced over the muscle. Four different surgeons with varying levels of experience performed the cranioplasty procedures on the patients in this study.

A postoperative subcutaneous drainage catheter was inserted, and prophylactic antibiotics were applied preoperatively and postoperatively in all patients.

\section{Statistical Analysis}

Candidate risk factors were compared between the SSI and non-SSI groups, and the bone flap resorption and nonbone flap resorption groups with the Mann-Whitney U-test for continuous variables and Pearson's Chi-square test for categorical variables. Independent associations between risk factors and clinical outcomes were analyzed using binary logistic regression, and odds ratios (ORs), and their 95\% confidence intervals $(\mathrm{Cls})$ were calculated. All results were expressed as mean \pm standard deviation (SD). The threshold for statistical significance was set at $p<0.05$. Analyses were performed using the IBM Statistical Package for the Social Sciences, version 18.0 (SPSS Inc., Chicago, IL, USA).

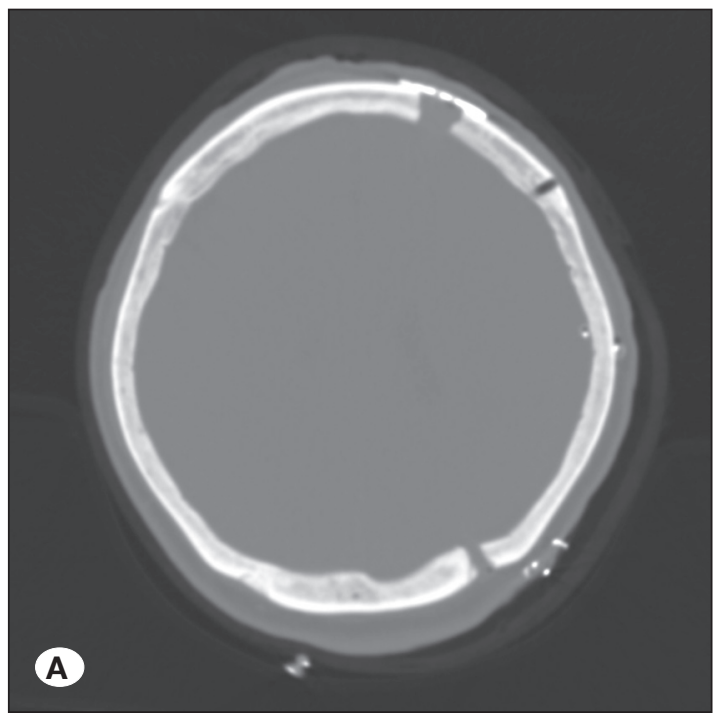

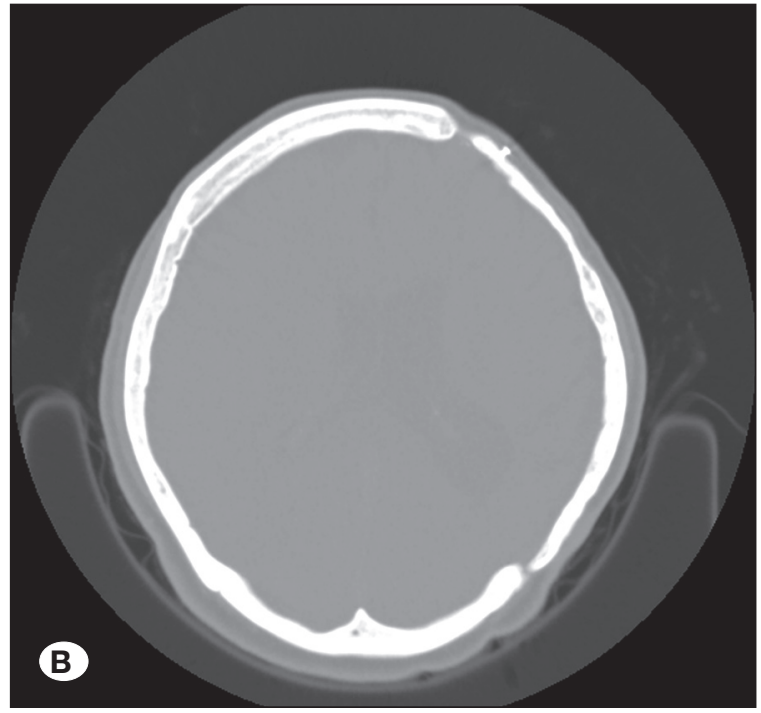

Figure 1: Changes in graft side bone thickness. Serial CT scans taken immediately after cranioplasty (A), and follow- up brain CT scans (B) show changes in graft side bone thickness. 


\section{RESULTS}

Basic data

Mean patient age was $43.1 \pm 15.0$ years and there were 40 (70.2\%) men and 17 (29.8\%) women. Forty patients $(70.2 \%)$ had a history of smoking and five patients $(8.8 \%)$ had diabetes mellitus. Thirty-six patients $(63.2 \%)$ had decompressive craniectomy for trauma, 7 (12.3\%) had cerebral infarctions, $10(17.6 \%)$ had subarachnoid hemorrhages, $3(5.3 \%)$ had intracerebral hemorrhages and $1(1.6 \%)$ had a tumor. The mean time from craniectomy to cranioplasty was $135.7 \pm 80.1$ days and the mean operative time was $160 \pm 46.8$ minutes. Five patients $(8.8 \%)$ underwent bilateral cranioplasty. Thirteen patients $(24.6 \%)$ had resection of the temporalis muscle during the craniectomy. These basic data are summarized in Table I. Patients were followed for a median of 43 months (range 2-108 months).

\section{Incidence of SSI}

SSI developed in 7 (12.3\%) out of 57 patients. In these cases, we performed an additional surgery for bone flap removal. Among these patients, one culture was negative. Of the 6 other patients, 4 were infected with Staphylococcus aureus (S. aureus) and one patient each was infected with Pseudomonas aeruginosa (P. aeruginosa) and Klebsiella oxytoca (K. oxytoca).
There were no statistically significant associations between SSI and age, gender, medical co-morbidity (diabetes mellitus, smoking), causes of craniectomy, operation time and bilateral craniectomy. However, there were statistically significant associations between SSI and time from craniectomy to cranioplasty ( $p=0.002$, Mann-Whitney $U$ test) and previous temporalis muscle resection $(p=0.021$, Pearson's Chi-square test) (Table I).

Univariate analysis revealed that, among the candidate factors, a decreased interval time from craniectomy to cranioplasty (OR $0.926,95 \% \mathrm{Cl} 0.868-0.989, \mathrm{p}=0.022)$ and previous temporalis muscle resection (OR 6.074, 95\% Cl 1.153-32.001, $\mathrm{p}=0.033$ ) were statistically correlated with patients who had SSI (Table II). These factors were also independently associated with SSI (time from craniectomy to cranioplasty: OR $0.901,95 \% \mathrm{Cl}$ $0.826-0.982, p=0.018$; previous temporalis muscle resection: OR 11.607, 95\% CI 1.155-116.590, p=0.037) (Table III).

\section{Incidence of Bone Flap Resorption}

We did not include patients in the bone flap resorption cohort for whom bone flap removal was caused by surgical site infection. Bone flap resorption was observed in 12 (24.0\%) out of 50 patients.

Table I: Characteristics of Cranioplasty Patients with or without Surgical Site Infections

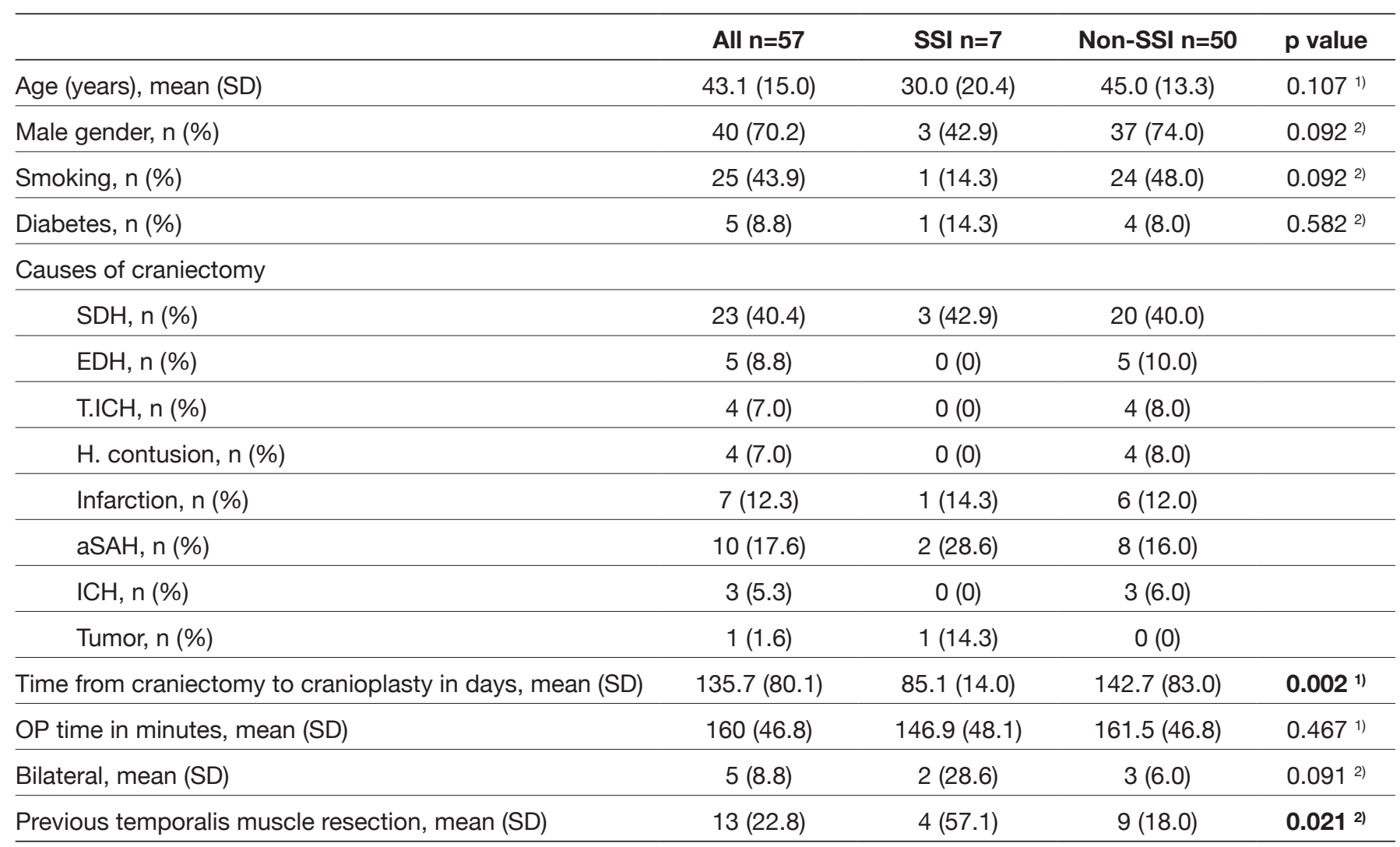

SSI: Surgical site infection, SDH: Subdural hematoma, EDH: Epidural hematoma, T.ICH: Traumatic intracranial hematoma, $\boldsymbol{H}$. contusion: Hemorrhagic contusion, aSAH: Aneurysmal subarachnoid hemorrhage, ICH: Intracranial hematoma, OP: Operation.

1) Mann-Whitney U-test. 2) Pearson's Chi-square test. 
There were no statistically significant associations between bone flap resorption and age, gender, medical comorbidity (diabetes mellitus, smoking), causes of craniectomy, the time from craniectomy to cranioplasty, operation time and bilateral craniectomy. However, there was a statistically significant association between bone flap resorption rate and previous temporalis muscle resection ( $\mathrm{p}=0.001$, Pearson's Chi-square test) (Table IV).

Univariate analysis revealed that previous temporalis muscle resection (OR 11.667, 95\% Cl 2.276-59.798, $\mathrm{p}=0.003$ ) was statistically associated with patients who had bone flap resorption (Table V).

Table II: Risk Factors for Surgical Site Infection Using Univariate Analysis

\begin{tabular}{lccc}
\hline \multirow{2}{*}{ Clinical characteristics } & \multicolumn{2}{c}{ Univariate study } \\
\cline { 2 - 4 } & OR & $\mathbf{9 5 \%}$ Cl for OR & p value \\
\hline Time from craniectomy to cranioplasty in days & 0.926 & $0.868-0.989$ & $\mathbf{0 . 0 2 2}$ \\
\hline Previous temporalis muscle resection & 6.074 & $1.153-32.001$ & $\mathbf{0 . 0 3 3}$ \\
\hline
\end{tabular}

Table III: Risk Factors for Surgical Site Infection Using Multivariate Analysis

\section{Clinical characteristics}

Time from craniectomy to cranioplasty in days

Previous temporalis muscle resection
Multivariate study

95\% Cl for OR p value

OR

$0.826-0.982$

0.018

11.607

0.037

Table IV: Characteristics of Cranioplasty Patients with or without Bone Flap Resorption

\begin{tabular}{|c|c|c|c|c|}
\hline & All $n=50$ & $\begin{array}{l}\text { Bone resorption } \\
n=12\end{array}$ & $\begin{array}{c}\text { No bone } \\
\text { resorption } n=38\end{array}$ & $\mathrm{p}$ value \\
\hline Age (years), mean (SD) & $45.0(13.3)$ & $46.6(17.2)$ & $44.4(12.0)$ & $0.540^{1)}$ \\
\hline Male sex, n (\%) & $37(74.0)$ & $10(83.3)$ & $27(71.1)$ & $0.398^{2)}$ \\
\hline Smoking, n (\%) & $24(48.0)$ & $6(50.0)$ & $18(47.4)$ & $0.874^{2)}$ \\
\hline Diabetes, n (\%) & $4(8.0)$ & $1(8.3)$ & $3(7.9)$ & $0.961^{2)}$ \\
\hline \multicolumn{5}{|l|}{ Causes of craniectomy } \\
\hline $\mathrm{SDH}, \mathrm{n}(\%)$ & $20(40.0)$ & $4(33.3)$ & $16(42.1)$ & \\
\hline $\mathrm{EDH}, \mathrm{n}(\%)$ & $5(10.0)$ & $2(16.7)$ & $3(7.9)$ & \\
\hline T.ICH, n (\%) & $4(8.0)$ & $2(16.7)$ & $2(5.3)$ & \\
\hline H. contusion, $\mathrm{n}(\%)$ & $4(8.0)$ & $1(8.3)$ & $3(7.9)$ & \\
\hline Infarction, n (\%) & $6(12.0)$ & $2(16.7)$ & $4(10.5)$ & \\
\hline aSAH, n (\%) & $8(16.0)$ & $1(8.3)$ & $7(18.4)$ & \\
\hline $\mathrm{ICH}, \mathrm{n}(\%)$ & $3(6.0)$ & $0(0)$ & $3(7.9)$ & \\
\hline Tumor, n (\%) & $0(0)$ & $0(0)$ & $0(0)$ & \\
\hline Time from craniectomy to cranioplasty in days, mean (SD) & $142.7(83.0)$ & $124.2(74.3)$ & $148.6(85.6)$ & $0.149^{1)}$ \\
\hline OP time in minutes, mean (SD) & $161.5(46.8)$ & $167.3(50.6)$ & $159.7(46.1)$ & $0.625^{1)}$ \\
\hline Bilateral, mean (SD) & $3(6.0)$ & $2(16.7)$ & $1(2.6)$ & $0.185^{2)}$ \\
\hline Previous temporalis muscle resection, mean (SD) & $9(18.0)$ & $6(50.0)$ & $3(7.9)$ & $0.001^{2)}$ \\
\hline
\end{tabular}

SDH: subdural hematoma, EDH: epidural hematoma, T.ICH: traumatic intracranial hematoma, $\boldsymbol{H}$. contusion: hemorrhagic contusion, aSAH: aneurysmal subarachnoid hemorrhage, ICH: intracranial hematoma, OP: operation.

1) Mann-Whitney U-test.

2) Pearson's Chi-square test. 
Table V: Risk Factors for Bone Flap Resorption Using Univariate Analysis

\begin{tabular}{lccc}
\hline \multirow{2}{*}{ Clinical characteristics } & \multicolumn{2}{c}{ Univariate study } \\
\cline { 2 - 4 } Previous temporalis muscle resection & OR & $\mathbf{9 5 \%}$ Cl for OR & p value \\
\hline
\end{tabular}

\section{DISCUSSION}

In the present study, 7 (12.3\%) of 57 patients who underwent cranioplasty after decompressive craniectomy underwent bone flap removal surgery due to surgical site infection. Except for these seven patients, twelve $(24.0 \%)$ out of 50 patients had bone flap resorption. Patients with SSI had a shorter time from craniectomy to cranioplasty and had a higher rate of removal of the temporalis muscle at the time of craniectomy than those who did not have infections. Patients who had bone flap resorption included a higher proportion of patients who underwent removal of the temporalis muscle than who did not have bone flap resorption.

\section{SSI}

Surgical site infection is a serious complication that can cause serious morbidities for patients. The incidence of SSI requiring removal of implanted bone and long-term intravenous antibiotic administration has been reported to be between $2-20 \%(2,11,26)$. At some institutions, the removed bone flaps are routinely checked for bacterial contamination prior to storage. Chiang et al., found that the frequency of SSI did not increase after implanting bone flaps contaminated with microorganisms such as $P$. acnes, coagulase-negative Staphylococci, and S. aureus (7). However, the risk of SSI in implanted bone flaps with positive bacterial cultures prior to preservation has not been determined. We do not know if the bone flaps were contaminated before cryopreservation, since bacteriological examination of the bone flap prior to preservation has not been implemented as a routine practice in our department.

So far, studies to clarify the risk factors for these infections have continued. Although various risk factors have been described in many reports, the most common risk factor was long operation time $(16,17,25)$. However, there were differences in the criteria for long operation time in these reports. According to Lee et al., the infection rate significantly increased when the operation time was longer than 200 minutes (17). In our study, the relationship between operation time and infection rate was not significant, possibly due to the limited number of patients.

The relationship between the interval of decompressive craniectomy and cranioplasty and SSI has been reported in several papers. According to Nagayama et al., there was a statistically significant difference between the two groups (20). The interval between surgeries was shorter in patients with SSI (average of 31.1 days in the SSI group and 54.9 days in the non-SSI group). In contrast, Matsuno et al. (19), reported that SSI patients had a significantly longer interval between surgeries. The average interval between surgeries was 108.1 days in patients with SSI and 74.0 days in patients without SSI. However, Bender et al., reported that the interval between operations did not increase the incidence of other complications including postoperative infection (3). In our study, although a limited number of patients were affected, a shorter time interval between operations was correlated with a higher infection rate. This is thought to be due to an increased risk of infection from the interruption of normal wound recovery and wound angiogenesis with a short duration between craniectomy and cranioplasty (6).

During decompressive craniectomy, occasionally the temporalis muscle and fascia occlude the external herniation of the edematous brain and interfere with effective decompression. Thus, some surgeons may resect the temporalis muscle and fascia during decompressive craniectomy (22). This temporalis muscle and fascia resection procedure is sometimes performed at our hospital. However, resection of the temporalis muscle requires coagulation of the middle temporal artery originating from the superficial temporal artery and anterior and posterior temporal arteries originating from the internal maxillary artery (15). This process leads to poor vascularization of the pericranial flap and may cause wound disruption and infection (16). In our study, temporalis muscle resection was performed in $22.8 \%$ (13/57) of patients and there was a statistically significant difference when SSI and non-SSI patients were compared $(57.1 \%$ vs. $18.0 \% ; p=0.021)$. These results suggest that it is better not to perform temporalis muscle resection if possible.

\section{Bone Flap Resorption}

Several authors have reported the risk factors for bone flap resorption after cranioplasty with autologous cryopreserved bone $(1,5,12,13,23)$. Some studies have reported an increased risk of bone flap resorption in very young children $(12,23)$. However, in our patient cohort, only 2 of the 50 patients were young (15 and 16 years old), and statistical significance was not confirmed.

It has also been reported that bone fragmentation or cranioplasty performed within 2 months after craniectomy was a risk factor for bone flap resorption $(10,14,24)$. Analysis of these parameters in our cohort revealed no fragmented bone flaps. Also, in our cohort, the mean interval between craniectomy and cranioplasty was 124 days, which was longer than 2 months. Based on our findings, a short interval between craniectomy and cranioplasty did not seem to affect bone resorption.

Cranioplasty with autologous cryopreserved bone is associated with bone resorption rates. Bone flap non-viability may be a contributing factor. According to Bhaskar et al. (4), no cell growth was observed in any tissue when cell culture was per- 
formed on skull bone stored at $-30^{\circ} \mathrm{C}$ for more than 6 months. However, there may be some limitations in this study since it was an in vitro study.

On the other hand, Lu et al., performed cranioplasty using cryopreserved bone stored at $-80^{\circ} \mathrm{C}$ for 63 to 289 days (mean 111.7 days) in 16 patients, followed by 99Tc m-methylene diphosphonate (MDP) bone imaging (18). They confirmed the survival and regeneration of transplanted bone fragments (18). Based on these two results, autologous cryopreserved bones are not always non-viable.

Several studies reported that the regeneration of implanted bone fragments was achieved by introducing new blood vessels and osteoblasts into transplanted bone fragments at the normal skull bone edge $(18,23)$. Therefore, when performing the cranioplasty, the soft tissue at the edge of the cranial bone window should be sufficiently removed to expose the bone window edge and promote the formation of a new bone surface between the edge of the transplanted skull and the bone window edge. This will prevent bone resorption as much as possible. Sufficiently exposing the bone window edge for this purpose is standard procedure at our hospital.

In contrast to other studies, we found that the rate of bone resorption was higher in patients who had the temporalis muscle resected. This may be due to coagulation of the middle temporal artery originating from the anterior and posterior temporal arteries and the superficial temporal artery. The major blood supply to the calvaria is provided by the middle meningeal artery and its branches. The majority of the outer surface of the craniofacial skeleton is supplied by tiny perforators from the overlying periosteum (8). However, most craniectomy patients undergo coagulation of the middle meningeal artery during surgery. In addition, coagulation of the above-mentioned blood vessels leads to poor vascularization of the pericranium, thus promoting bone resorption. However, the exact cause of this finding may require more experimental research.

\section{Limitations}

Our study is a retrospective analysis and can suffer from unexpected defects related to loss of patient information. Various neurosurgeons and heterogeneous patients, including the use of various surgical techniques and surgical indications, may have influenced the results. Also, because the sample size is small, we cannot make firm conclusions about the risks associated with SSI and bone flap resorption.

Despite these limitations, our study has clear clinical significance and suggests that preserving the temporalis muscle helps to reduce SSI and bone flap resorption, the most serious complications of cranioplasty with autologous bone.

\section{CONCLUSION}

Postoperative infection and bone flap resorption are the most frequent complications associated with the reimplantation of autologous cryopreserved bone after decompressive craniectomy. The risk of these complications particularly increased after previous temporalis muscle resection. Based on this finding, we believe that preservation of the temporalis muscle may help decrease postoperative complications after autologous cranioplasty.

\section{ACKNOWLEDGEMENT}

This study was conducted by 2016 Korea University Ansan Hospital R\&D support project through the support of Vice President for Medical Affairs of Korea University special research funds (NO. 1613701).

\section{REFERENCES}

1. Asano Y, Ryuke $Y$, Hasuo M, Simosawa S: Cranioplasty using cryopreserved autogenous bone. No To Shinkei 45:11451150, 1993 (In Japanese)

2. Beauchamp KM, Kashuk J, Moore EE, Bolles G, Rabb C, Seinfeld J, Szentirmai O, Sauaia A: Cranioplasty after postinjury decompressive craniectomy: Is timing of the essence? J Trauma 69: 270-274, 2010

3. Bender A, Heulin S, Rohrer S, Mehrkens JH, Heidecke V, Straube A, Pfefferkorn T: Early cranioplasty may improve outcome in neurological patients with decompressive craniectomy. Brain Inj 27:1073-1079, 2013

4. Bhaskar IP, Yusheng L, Zheng M, Lee GY: Autogenous skull flaps stored frozen for more than 6 months: Do they remain viable? J Clin Neurosci 18:1690-1693, 2011

5. Cheng $\mathrm{CH}$, Lee HC, Chen CC, Cho DY, Lin HL: Cryopreservation versus subcutaneous preservation of autologous bone flaps for cranioplasty: Comparison of the surgical site infection and bone resorption rates. Clin Neurol Neurosurg 124: 85-89, 2014

6. Cheng YK, Weng HH, Yang JT, Lee MH, Wang TC, Chang $\mathrm{CN}$ : Factors affecting graft infection after cranioplasty. J Clin Neurosci 15:1115-1119, 2008

7. Chiang HY, Steelman VM, Pottinger JM, Schlueter AJ, Diekema DJ, Greenlee JD, Howard MA 3rd, Herwaldt LA: Clinical significance of positive cranial bone flap cultures and associated risk of surgical site infection after craniotomies or craniectomies. J Neurosurg 114:1746-1754, 2011

8. Cutting CB, McCarthy JG, Berenstein A: Blood supply of the upper craniofacial skeleton: The search for composite calvarial bone flaps. Plast Reconstr Surg 74:603-610, 1984

9. Diedler J, Sykora M, Blatow M, Juttler E, Unterberg A, Hacke W: Decompressive surgery for severe brain edema. J Intensive Care Med 24:168-178, 2009

10. Dunisch P, Walter J, Sakr Y, Kalff R, Waschke A, Ewald C: Risk factors of aseptic bone resorption: A study after autologous bone flap reinsertion due to decompressive craniotomy. J Neurosurg 118:1141-1147, 2013

11. Gooch MR, Gin GE, Kenning TJ, German JW: Complications of cranioplasty following decompressive craniectomy: Analysis of 62 cases. Neurosurg Focus 26: E9, 2009

12. Grossman N, Shemesh-Jan HS, Merkin V, Gideon M, Cohen A: Deep-freeze preservation of cranial bones for future cranioplasty: Nine years of experience in Soroka University Medical Center. Cell Tissue Bank 8:243-246, 2007 
13. Hancock DO: The fate of replaced bone flaps. J Neurosurg 20: 983-984, 1963

14. Honeybul S, Ho KM: How "successful" is calvarial reconstruction using frozen autologous bone? Plast Reconstr Surg 130:1110-1117, 2012

15. Kadri PA, Al-Mefty O: The anatomical basis for surgical preservation of temporal muscle. J Neurosurg 100:517-522, 2004

16. Kim H, Sung SO, Kim SJ, Kim SR, Park IS, Jo KW: Analysis of the factors affecting graft infection after cranioplasty. Acta Neurochir (Wien) 155:2171-2176, 2013

17. Lee $\mathrm{CH}$, Chung YS, Lee SH, Yang HJ, Son YJ: Analysis of the factors influencing bone graft infection after cranioplasty. J Trauma Acute Care Surg 73:255-260, 2012

18. Lu Y, Hui G, Liu F, Wang Z, Tang Y, Gao S: Survival and regeneration of deep-freeze preserved autologous cranial bones after cranioplasty. Br J Neurosurg 26:216-221, 2012

19. Matsuno A, Tanaka H, Iwamuro H, Takanashi S, Miyawaki $\mathrm{S}$, Nakashima M, Nakaguchi H, Nagashima T: Analyses of the factors influencing bone graft infection after delayed cranioplasty. Acta Neurochir (Wien) 148:535-540, 2006

20. Nagayama K, Yoshikawa G, Somekawa K, Kohno M, Segawa H, Sano K, Shiokawa Y, Saito I: Cranioplasty using the patient's autogenous bone preserved by freezing-an examination of post-operative infection rates. No Shinkei Geka 30:165-169, 2002

21. Otani N, Takasato Y, Masaoka H, Hayakawa T, Yoshino Y, Yatsushige H, Miyawaki H, Sumiyoshi K, Chikashi A, Takeuchi S, Suzuki G: Surgical outcome following decompressive craniectomy for poor-grade aneurysmal subarachnoid hemorrhage in patients with associated massive intracerebral or Sylvian hematomas. Cerebrovasc Dis 26:612-617, 2008
22. Park J, Kim E, Kim GJ, Hur YK, Guthikonda M: External decompressive craniectomy including resection of temporal muscle and fascia in malignant hemispheric infarction. $J$ Neurosurg 110:101-105, 2009

23. Prolo DJ, Burres KP, McLaughlin WT, Christensen AH: Autogenous skull cranioplasty: fresh and preserved (frozen), with consideration of the cellular response. Neurosurgery 4: 18-29, 1979

24. Schuss P, Vatter H, Oszvald A, Marquardt G, Imohl L, Seifert V, Guresir E: Bone flap resorption: Risk factors for the development of a long-term complication following cranioplasty after decompressive craniectomy. J Neurotrauma 30:91-95, 2013

25. Sundseth J, Sundseth A, Berg-Johnsen J, Sorteberg W, Lindegaard KF: Cranioplasty with autologous cryopreserved bone after decompressive craniectomy: Complications and risk factors for developing surgical site infection. Acta Neurochir (Wien) 156:805-811; discussion 811, 2014

26. Tokoro K, Chiba Y, Tsubone K: Late infection after cranioplastyReview of 14 cases. Neurol Med Chir (Tokyo) 29:196-201, 1989

27. Vahedi K, Hofmeijer J, Juettler E, Vicaut E, George B, Algra A, Amelink GJ, Schmiedeck P, Schwab S, Rothwell PM, Bousser MG, van der Worp HB, Hacke W, Decimal D, investigators H: Early decompressive surgery in malignant infarction of the middle cerebral artery: A pooled analysis of three randomised controlled trials. Lancet Neurol 6:215-222, 2007

28. Yamada H, Sakai N, Takada M, Ando T, Kagawa Y: Cranioplasty utilizing a preserved autogenous bone flap coated with acrylic resin. Acta Neurochir (Wien) 52:273-280, 1980 